\title{
A GENERAL NONLINEAR VERSION OF ROTH'S THEOREM ON THE REAL LINE
}

\section{Xiang Li, Dunyan Yan, Haixia Yu And Xingsong Zhang*}

Abstract. Let $N>1$ be a real number and $\varepsilon>0$ be given. In this paper, we will prove that, for a measurable subset $S$ of $[0, N]$ with positive density $\varepsilon$, there must be patterns of the form $(x, x+t, x+\gamma(t))$ such that

$$
x, x+t, x+\gamma(t) \in S,
$$

where $\gamma$ is convex and has some curvature constraints, $t>\delta(\varepsilon, \gamma) \gamma^{-1}(N)$ and $\delta(\varepsilon, \gamma)$ is a positive constant depending only on $\varepsilon$ and $\gamma, \gamma^{-1}$ is the inverse function of $\gamma$. Our result extends Bourgain's result [2] to the general curve $\gamma$. We use Bourgain's energy pigeonholing argument and Li's $\sigma$-uniformity argument.

Mathematics subject classification (2020): Primary 42B20; Secondary 05D10.

Keywords and phrases: Roth's theorem, convex curve, stationary phase method, $\sigma$-uniformity.

\section{REFERENCES}

[1] V. Bergelson and A. Leibman, Polynomial extensions of van der Waerden's and Szemerédi's theorems, J. Amer. Math. Soc. 9 (1996), no. 3, 725-753.

[2] J. Bourgain, A nonlinear version of Roth's theorem for sets of positive density in the real line, J. Analyse Math. 50 (1988), 169-181.

[3] J. Bourgain, A Szemerédi type theorem for sets of positive density in $R^{k}$, Israel J. Math. 54 (1986), no. 3, 307-316.

[4] J. Bourgain, M.-C. Chang, Nonlinear Roth type theorems in finite fields, Israel J. Math. 221 (2017), no. 2, 853-867.

[5] A.-P. CALDERón, Cauchy integrals on Lipschitz curves and ralated operators, Proc. Nat. Acad. Sci. U.S.A. 74 (1977), no. 4, 1324-1327.

[6] A. Carbery, M. Christ, J. Vance, S. Wainger And D. Watson, Operators associated to flat plane curves: $L^{p}$ estimates via dilation methods, Duke Math. J. 59 (1989), no. 3, 675-700.

[7] A. CARbery, J. VAnce, S. WAinger And D. Watson, The Hilbert transform and maximal function along flat curves, dilations, and differential equations, Amer. J. Math. 116 (1994), no. 5, $1203-$ 1239.

[8] H. Carlsson, M. Christ, A. Cordoba, J. Duoandikoetxea, J. L. Rubio de Francia, J. VANCE, S. WAINGER AND D. WeINBERG, $L^{p}$ estimates for maximal functions and Hilbert transforms along flat convex curves in $\mathbb{R}^{2}$, Bull. Amer. Math. Soc. (N.S.) 14 (1986), no. 2, 263-267.

[9] M. Christ, X. LI, T. TAO AND C. ThIELE, On multilinear oscillatory integrals, nonsingular and singular, Duke Math. J. 130 (2005), no. 2, 321-351.

[10] M. Christ, A. Nagel, E. M. Stein And S. Wainger, Singular and maximal Radon transforms: analysis and geometry, Ann. of Math. (2) 150 (1999), no. 2, 489-577.

[11] B. Cook, Á. Magyar And M. Pramanik, A Roth-type theorem for dense subsets of $\mathbb{R}^{d}$, Bull. Lond. Math. Soc. 49 (2017), no. 4, 676-689.

[12] D. Dong, X. Li AND W. SAWIn, Improved estimates for polynomial Roth type theorems in finite fields, arXiv:1709.00080, 2017.

[13] P. Durcik, S. Guo And J. Roos, A polynomial Roth theorem on the real line, Trans. Amer. Math. Soc. 371 (2019), no. 10, 6973-6993. 
[14] P. ERdős AND P. Turań, On Some Sequences of Integers, J. London Math. Soc. 11 (1936), no. 4, 261-264.

[15] E. B. Fabes And N. M. Rivière, Singular integrals with mixed homogeneity, Studia Math. 27 (1966), 19-38.

[16] R. Fraser, S. Guo And M. Pramanik, Polynomial Roth theorems on sets of fractional dimensions, arXiv:1904.11123, 2019.

[17] H. FURSTENBERG, Ergodic behavior of diagonal measures and a theorem of Szemerédi on arithmetic progressions, J. Analyse Math. 31 (1977), 204-256.

[18] H. FuRSTENBERG AND Y. KATZNELSON, An ergodic Szemerédi theorem for commuting transformations, J. Analyse Math. 34 (1978), 275-291 (1979).

[19] W. T. Gowers, A new proof of Szemerédi theorem, Geom. Funct. Anal. 11 (2001), no. 3, 465-588.

[20] W. T. GowERs, A new proof of Szemerédi theorem for arithmetic progressions of length four, Geom. Funct. Anal. 8 (1998), no. 3, 529-551.

[21] B. GReEn, On arithmetic structures in dense sets of integers, Duke Math. J. 114 (2002), no. 2, $215-$ 238.

[22] B. GReEn AND T. TAO, The primes contain arbitrarily long arithmetic progressions, Ann. of Math. (2) 167 (2008), no. 2, 481-547.

[23] J. Guo And L. XiaO, Bilinear Hilbert transforms associated with plane curves, J. Geom. Anal. 26 (2016), no. 2, 967-995.

[24] L. HörmANDER, Oscillatory integrals and multipliers on $F L^{p}$, Ark. Mat. 11 (1973), 1-11.

[25] B. F. Jones, A class of singular integrals, Amer. J. Math. 86 (1964), 441-462.

[26] M. T. Lacey And C. Thiele, $L^{p}$ estimates on the bilinear Hilbert transform for $2<p<\infty$, Ann. of Math. (2) 146 (1997), no. 3, 693-724.

[27] M. T. Lacey And C. Thiele, On Calderón's conjecture, Ann. of Math. (2) 149 (1999), no. 2, 475-496.

[28] J. LI AND H. YU, $L^{p}$ boundedness of Hilbert transforms associated with variable plane curves, arXiv: 1806.08589, 2018.

[29] X. LI, Bilinear Hilbert transforms along curves I: The monomial case, Anal. PDE 6 (2013), no. 1, 197-220.

[30] X. Li AND L. XIAO, Uniform estimates for bilinear Hilbert transforms and bilinear maximal functions associated to polynomials, Amer. J. Math. 138 (2016), no. 4, 907-962.

[31] V. LIE, On the boundedness of the bilinear Hilbert transform along "non-flat" smooth curves, Amer. J. Math. 137 (2015), no. 2, 313-363.

[32] V. LIE, On the boundedness of the bilinear Hilbert transform along "non-flat" smooth curves. The Banach triangle case ( $\left.L^{r}, 1 \leqslant r<\infty\right)$, Rev. Mat. Iberoam. 34 (2018), no. 1, 331-353.

[33] A. Nagel, J. VAnce, S. Wainger And D. Weinberg, Hilbert transforms for convex curves, Duke Math. J. 50 (1983), no. 3, 735-744.

[34] K. F. Roth, On certain sets of integers, J. London Math. Soc. 28 (1953), 104-109.

[35] E. M. Stein, Harmonic Analysis: Real-Variable Methods, Orthogonality, and Oscillatory Integrals, Princeton Mathematical Series 43, Monographs in Harmonic Analysis III, Princeton University Press, Princeton, NJ, 1993.

[36] E. SZEMERÉDI, On sets of integers containing no four elements in arithmetic progression, Acta Math. Acad. Sci. Hungar. 20 (1969), 89-104.

[37] E. SZEMERÉDI, On sets of integers containing no $k$ elements in arithmetic progression, Acta Arith. 27 (1975), 199-245.

[38] T. TAO AND T. ZIEGLER, A multi-dimensional Szemerédi theorem for the primes via a correspondence principle, Israel J. Math. 207 (2015), no. 1, 203-228.

[39] T. TAO AND T. ZIEgleR, The primes contain arbitrarily long polynomial progressions, Acta Math. 201 (2008), no. 2, 213-305. 